We would expect that a bunch of rays focused at a point should 5 minutes later refocus in a ring some $2000 \mathrm{~km}$ in radius.

$R$. Michard. I am ready to assume that high frequency sound waves can indeed be trapped as described by Dr Kahn. But it seems that such considerations cannot explain the observed oscillations of the solar atmosphere: in the observations points of maximum correlation are not displaced.

F. Kahn. Perhaps one can relate the observed Doppler shifts with these focused waves as follows. When waves are well focused in a particular volume element, this leads to an increase in the energy content of the gas, which then becomes more buoyant and tends to rise. In between focusing periods the element must fall again: perhaps this explains the observed anticorrelation.

$P$. A. Sturrock. An alternative explanation of the 5 -minute resonance is that the group velocity of acoustic waves is zero for this frequency.

A. H. Cook. Dr Kahn's proposed mechanism for producing vertical motions is similar to Longuet-Higgins mechanism for generating microseism to vertical pressures on the sea-flow from interference of progressive waves. If so, there is a factor of 2 between the period of progressive waves and that of the vertical motions.

\title{
9. GENERATION AND PROPAGATION OF OSCILLATORY MOTIONS IN THE SOLAR ATMOSPHERE
}

\section{F. Meyer and H. U. Schmidt}

(High Altitude Observatory, Boulder, Colorado, and Sacramento Peak Observatory, Sunspot, New Mexico; on leave from Max-Planck-Institut für Astrophysik, Munich.)

It seems reasonable to assume that single photospheric granules are the proper sources of the periodic vertical motions in the solar atmosphere observed by Evans and Michard (I). A granule may be thought of as a small upwelling of photospheric matter which settles down to its normal position at the end of the lifetime of the visible granule. This lifetime is by no means negligible compared with the average periods or lifetimes of the observed coherent oscillations. This implies that we deal with an inhomogeneous boundary value problem, where the inhomogeneity at the boundary, i.e. the granule, influences the solution throughout the interesting range of time. Therefore a superposition of many modes fitting realistic initial and boundary values is needed. Such a superposition might easily explain the observed time lags of the oscillations in different spectral lines or heights. E.g. if we restrict ourselves to one dimension, i.e. to a purely vertical propagation of the waves, and if we assume an initially quiet isothermal atmosphere under gravitation, which is pushed from below by an upward moving piston beginning its movement at $t_{0}$, we get a very reasonable propagation of phases, though not of the amplitudes as was shown earlier (2). In this model the maxima and minima of the oscillating velocity at different heights exhibit clearly a decreasing vertical time lag approaching zero and an increasing period of time between subsequent maxima approaching the resonance value of 300 seconds.

The observed oscillations generally exhibit frequencies within the non-propagating range of the dispersion relation discussed by Moore and Spiegel (3). We believe that they are built up asymptotically by the neighboring propagating wave modes which can be excited by the 
boundary values. The granules with their small dimensions will not be able to excite the oscillatory modes near the upper critical period of about 300 seconds with the corresponding small values of the wave number at once. On the other hand, the resonance character of the dispersion relation lowers the group velocity in the neighborhood of the resonance and this provides means to build up non-propagating resonance oscillations asymptotically if the boundary condition contains a reasonable frequency spectrum in a reasonable order of phases. Furthermore the pure gravity waves of type IV described by Whitney (4) can arise and propagate throughout this frequency range.

But if we really deal with a three-dimensional propagation phenomenon, as we believe, how can the observed large horizontal scale of coherence ( 2 or 3 or even $5000 \mathrm{~km}$ ) be explained? The answer is that the wave most likely is observed where the local wave front is parallel to the slit of the spectrograph. This is the position nearest to the source and the length of coherence can be of the same order as the distance from the source especially so for the enlarged phase velocities in the neighborhood of the resonance.

To test these ideas some computations were carried out on an IBM I620 at Sacramento Peak Observatory. So far we have assumed isothermal disturbances in an isothermal atmosphere. Further we assume rotational symmetry around a vertical axis through the center of the exciting granule. The boundary condition at $z=0$ is a vertical displacement of the boundary during the lifetime of a granule and over a region of the size of a granule. We take both the functions of time and of radius as Gaussian. This rigid displacement implies total reflection which is justified by the inertia of the subphotospheric layers. As upper boundary we take the interface between chromosphere and corona. From the work of Bahng and Schwarzschild (5) one can draw the conclusion that this interface forms a fairly good reflector, at least as long as it is a smooth surface. We set the velocity divergence zero at this boundary to represent total reflection at a medium free of inertia. The initial condition is total rest at a time $t_{0}$ where the Gaussian disturbance at the lower boundary would have been smaller than the overall computation accuracy of about 0.5 per cent of the maximum disturbance.

We fulfill the boundary conditions by superposition of proper modes which contain Bessel functions for the radial dependence. This gives a time symmetric solution which does not yet fulfill the initial values. To enforce these we have to add a suitable superposition of proper modes of the homogeneous problem, i.e. for vanishing lower boundary values. This compensates the converging wave pattern for times preceeding the disturbance.

So far we use a temperature of $9000^{\circ}$, a chromospheric thickness of 6 scale heights or $2200 \mathrm{~km}$. The half-width of the radial Gaussian is $1500 \mathrm{~km}$ corresponding to a large granule. The vectors of displacement and velocity are computed at $10 \times 5$ grid points $450 \mathrm{~km}$ apart and for I I 5 time steps covering about 25 minutes. These computations are completed for three different time scales for the Gaussian disturbance. If we call the period which begins and ends with a perturbation of I per cent of its maximum value the lifetime of the exciting granule, these lifetimes are $4.4 ; 8.8$ and 16 minutes.

The I5-minute solution exhibits mainly a single rise and fall throughout the medium similar to the Gaussian boundary disturbance. The secondary maxima are smaller than 3 per cent, not in agreement with the observations. The $4 \cdot 4$-minute solution on the contrary shows increasing irregular oscillations with short periods in the range of 150 seconds also not in agreement with the observations. The 8.8 minute solution using the observed average for the lifetime of a granule (Bahng and Schwarzschild (6)) exhibits strong almost undamped oscillations with periods approaching 300 seconds from below and initially sonic vertical time lags vanishing as time goes on. All these features are in good agreement with the observations of Evans and Michard (r). This solution was presented at the Joint Discussion in form of a movie showing the time dependent displacements of all 50 grid points. 


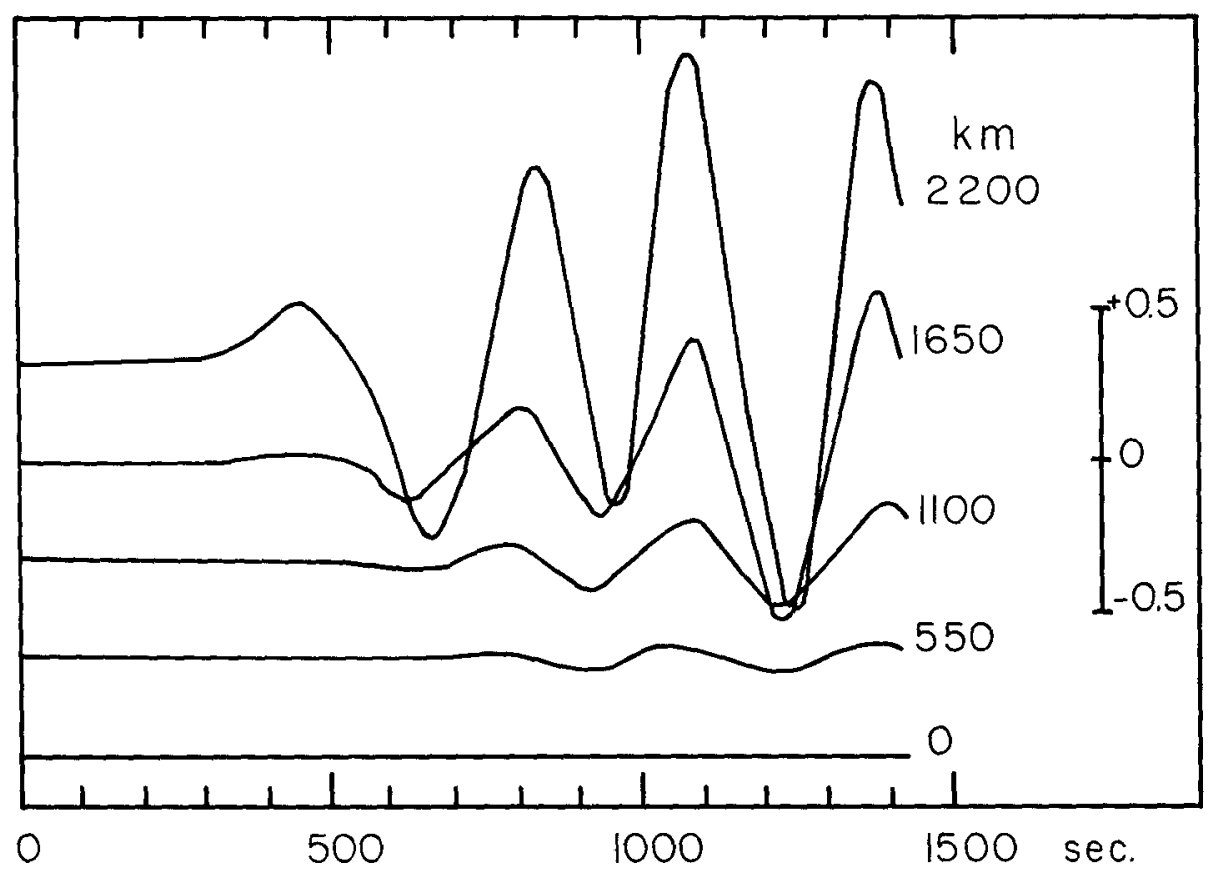

Fig. I. Vertical velocity as a function of time at different levels, $4000 \mathrm{~km}$ from the center. The scale at the right gives the velocity in units of the maximum velocity at the origin.

\section{REFERENCES}

I. Evans, J. W., Michard, R. Astrophys. F., 136, 493, 1962.

2. Schmidt, H. U., Zirker, J. B. Astrophys. F., 138, I310, 1963.

3. Moore, D. W., Spiegel, E. A. Astrophys. F., 139, 48, 1964.

4. Whitney, C. Smithson. Contr. Astrophys., 2, 365, I958.

5. Bahng, J. D., Schwarzschild, M. Astrophys. F., r37, 901, I963.

6. Bahng, J. D., Schwarzschild, M. Astrophys. F., 134, 312, I961.

\section{I0. WAVE MOTION AND THE STRUCTURE OF THE SOLAR CHROMOSPHERE \\ P. A. Sturrock \\ (Stanford University, California)}

Although the structure of the solar atmosphere depends to some extent on the magnetic field, it cannot be said to be highly sensitive to this field. This fact indicates that the nonthermal energy flux responsible for heating the chromosphere and corona is predominantly acoustic rather than magnetohydrodynamic.

If one considers that the energy propagation is in the form of acoustic waves, and takes note of the fact that the propagation characteristics of acoustic waves in a gravitating atmosphere 